\title{
Systems of Navier-Stokes Equations on Cantor Sets
}

\author{
Xiao-Jun Yang, ${ }^{1}$ Dumitru Baleanu, ${ }^{2,3,4}$ and J. A. Tenreiro Machado ${ }^{5}$ \\ ${ }^{1}$ Department of Mathematics and Mechanics, China University of Mining and Technology, Xuzhou Campus, Xuzhou, \\ Jiangsu 221008, China \\ ${ }^{2}$ Department of Mathematics and Computer Sciences, Faculty of Arts and Sciences, Cankaya University, 06530 Ankara, Turkey \\ ${ }^{3}$ Institute of Space Sciences, Magurele, Bucharest, Romania \\ ${ }^{4}$ Department of Chemical and Materials Engineering, Faculty of Engineering, King Abdulaziz University, P.O. Box 80204, \\ Jeddah 21589, Saudi Arabia \\ ${ }^{5}$ Department of Electrical Engineering, Institute of Engineering, Polytechnic of Porto, Rua Dr. Antonio Bernardino de Almeida, \\ 431, 4200-072 Porto, Portugal
}

Correspondence should be addressed to Xiao-Jun Yang; dyangxiaojun@163.com

Received 31 March 2013; Revised 3 June 2013; Accepted 10 June 2013

Academic Editor: Bashir Ahmad

Copyright ( $\odot 2013$ Xiao-Jun Yang et al. This is an open access article distributed under the Creative Commons Attribution License, which permits unrestricted use, distribution, and reproduction in any medium, provided the original work is properly cited.

We present systems of Navier-Stokes equations on Cantor sets, which are described by the local fractional vector calculus. It is shown that the results for Navier-Stokes equations in a fractal bounded domain are efficient and accurate for describing fluid flow in fractal media.

\section{Introduction}

The Navier-Stokes equations [1-3] are commonly used in describing motion of fluids in models relevant to weather, ocean currents, water flow in pipes, and so forth.

However, the turbulent flows may be of fractal character, and the smoothness required in the common problem modelled by the Navier-Stokes equations may be violated in fractal flows. Such distortions exist in both small-scale [4] and large-scale $[5,6]$ turbulent flows. In this context, Benzi et al. [7] investigated the scaling properties of turbulent flows while She and Leveque [8] stressed the attention on universal scaling laws in fully developed turbulence. Shraiman and Siggia also reported scalar turbulence [9], while the selfsimilarity in turbulent flows was analyzed by [10]. The fractal dimension of turbulent flows was pointed out in several studies [10-12], and some applications involving the NavierStokes equations were suggested [13-15] taking into account the multifractal nature of fully developed turbulence and chaotic systems [16]. In this context, the alpha model of turbulence $[17,18]$ and its applications to Navier Stokes have been discussed [19].

Based on fractional calculus theory [20-23] timefractional Navier-Stokes equation has been recently proposed by El-Shahed and Salem [24]. Several analytical solutions have been developed by the Adomian decomposition method [25] and the homotopy perturbation method [26].

Recently, the element of fractal arc length squared in fractal time-space was discussed [27]. Based on it, the Schrödinger equation [28], the heat conduction equation $[27,29]$, the wave equation $[27,30]$, and the diffusion equation on Cantor time-space [31] were suggested based on local fractional calculus theory [27, 32, 33]. Carpinteri and Sapora had reported diffusion equation on Cantor space [34]. Kolwankar and Gangal had proposed the Fokker-Planck equation on Cantor space [35].

In this paper we employ the local fractional vector calculus [27], which is set up on fractals, to model systems of Navier-Stokes equations on Cantor sets. The paper is organized as follows. In Section 2, a short introduction to local fractional vector calculus theory is given. Fractal kinematics, continuity equations, and constitutive equations for Newtonian fluid on Cantor sets are discussed in Section 3. Cauchy's equations of motion and mechanical energy equations on Cantor sets are investigated in Section 4. Systems of Navier-Stokes equations on Cantor sets with Cantorian coordinates systems are presented in Section 5. Finally, in Section 6, the conclusions are presented. 


\section{Mathematical Tool}

Local fractional vector form of function gives [27]

$$
\mathbf{r}(x)=M(x) \mathbf{e}_{1}^{\alpha}+N(x) \mathbf{e}_{2}^{\alpha}
$$

which is expressed by

$$
\mathbf{r}(x)=(M(x), N(x)) .
$$

For a function of three variables, the vector form can be written in the form [27]

$$
\mathbf{r}(x, y, z)=L(x, y, z) \mathbf{e}_{1}^{\alpha}+M(x, y, z) \mathbf{e}_{2}^{\alpha}+N(x, y, z) \mathbf{e}_{3}^{\alpha},
$$

or

$$
\mathbf{r}(x, y)=(L(x, y, z), M(x, y, z), N(x, y, z)) .
$$

Let $\mathbf{u}(x, y, z)=u(x, y, z) \mathbf{e}_{1}^{\alpha}+u_{2}(x, y, z) \mathbf{e}_{2}^{\alpha}+u_{3}(x, y, z) \mathbf{e}_{3}^{\alpha} \mathrm{a}$ local fractional vector field and $\varphi(x, y, z)$ be a local fractional scalar field, the computing rules of Hamilton operator are valid as follows [27].

(1) The local fractional gradient and Laplace operator of a local fractional scalar field are, respectively, defined as [27]

$$
\begin{gathered}
\nabla^{\alpha} \varphi=\frac{\partial^{\alpha} \varphi}{\partial x_{1}^{\alpha}} \mathbf{e}_{1}^{\alpha}+\frac{\partial^{\alpha} \varphi}{\partial x_{2}^{\alpha}} \mathbf{e}_{2}^{\alpha}+\frac{\partial^{\alpha} \varphi}{\partial x_{3}^{\alpha}} \mathbf{e}_{3}^{\alpha}, \\
\nabla^{2 \alpha} \varphi=\frac{\partial^{2 \alpha} \varphi}{\partial x_{1}^{2 \alpha}}+\frac{\partial^{2 \alpha} \varphi}{\partial x_{2}^{2 \alpha}}+\frac{\partial^{2 \alpha} \varphi}{\partial x_{3}^{2 \alpha}},
\end{gathered}
$$

where local fractional partial derivatives of high order are defined as [27, 31-33]

$$
\frac{\partial^{k \alpha}}{\partial x^{k \alpha}} f(x)=\overbrace{\frac{\partial^{\alpha}}{\partial x^{\alpha}} \cdots \frac{\partial^{\alpha}}{\partial x^{\alpha}}}^{k \text { times }} f(x) \text {. }
$$

(2) The local fractional divergence and curl of a local fractional vector field are written in the form $[27,31]$

$$
\begin{aligned}
\operatorname{div}^{\alpha} \mathbf{u}=\nabla^{\alpha} \cdot \mathbf{u}= & \frac{\partial^{\alpha} u_{1}}{\partial x_{1}^{\alpha}}+\frac{\partial^{\alpha} u_{2}}{\partial x_{2}^{\alpha}}+\frac{\partial^{\alpha} u_{3}}{\partial x_{3}^{\alpha}} \\
\operatorname{curl}^{\alpha} \mathbf{u}=\nabla^{\alpha} \times \mathbf{u}= & \left(\frac{\partial^{\alpha} u_{3}}{\partial x_{2}^{\alpha}}-\frac{\partial^{\alpha} u_{2}}{\partial x_{3}^{\alpha}}\right) \mathbf{e}_{1}^{\alpha} \\
& +\left(\frac{\partial^{\alpha} u_{1}}{\partial x_{3}^{\alpha}}-\frac{\partial^{\alpha} u_{3}}{\partial x_{1}^{\alpha}}\right) \mathbf{e}_{2}^{\alpha} \\
& +\left(\frac{\partial^{\alpha} u_{2}}{\partial x_{1}^{\alpha}}-\frac{\partial^{\alpha} u_{1}}{\partial x_{2}^{\alpha}}\right) \mathbf{e}_{3}^{\alpha} .
\end{aligned}
$$

(3) The following equations are valid only in Cantor coordinates [27]:

$$
\begin{gathered}
\nabla^{\alpha}(\mathbf{u v})=\left(\nabla^{\alpha} \mathbf{u}\right) \mathbf{v}+\mathbf{u} \nabla^{\alpha} \mathbf{v}, \\
\nabla^{2 \alpha} \mathbf{A}=\nabla^{\alpha}\left(\nabla^{\alpha} \cdot \mathbf{A}\right)-\nabla^{\alpha} \times\left(\nabla^{\alpha} \times \mathbf{A}\right), \\
\mathbf{A} \cdot \nabla^{\alpha} \mathbf{A}=\nabla^{\alpha}\left(\frac{\mathbf{A} \cdot \mathbf{A}}{2}\right)-\left(\nabla^{\alpha} \times \mathbf{A}\right) \times \mathbf{A},
\end{gathered}
$$

where $\nabla^{2 \alpha}=\nabla^{\alpha} \cdot \nabla^{\alpha}$ is local fractional Laplace operator [27].

The local fractional line integral of the function $\mathbf{u}\left(x_{P}, y_{P}, z_{P}\right)$ in the local fractional vector form

$$
\begin{aligned}
\mathbf{u}\left(x_{P}, y_{P}, z_{P}\right)= & u_{1}\left(x_{P}, y_{P}, z_{P}\right) \mathbf{e}_{1}^{\alpha}+u_{2}\left(x_{P}, y_{P}, z_{P}\right) \mathbf{e}_{2}^{\alpha} \\
& +u_{3}\left(x_{P}, y_{P}, z_{P}\right) \mathbf{e}_{3}^{\alpha}
\end{aligned}
$$

along a fractal line $l^{\alpha}$ is written as [27]

$$
\int_{l^{(\alpha)}} \mathbf{u}\left(x_{P}, y_{P}, z_{P}\right) \cdot d \mathbf{l}^{\alpha}=\lim _{N \rightarrow \infty} \sum_{P=1}^{N} \mathbf{u}\left(x_{P}, y_{P}, z_{P}\right) \cdot \Delta \mathbf{l}_{P}^{\alpha},
$$

where for $P=1,2, \ldots, N$ and $N$ elements of line $\Delta \mathbf{l}_{P}^{\alpha}$, it is required that all $\left|\Delta l_{P}^{\alpha}\right| \rightarrow 0$ as $N \rightarrow \infty$.

The local fractional surface integral of the given function (9) across a surface $S^{(\beta)}$ is defined as [27]

$$
\begin{aligned}
& \iint_{S^{(\beta)}} \mathbf{u}\left(x_{P}, y_{P}, z_{P}\right) \cdot d \mathbf{S}^{(\beta)} \\
& \quad=\lim _{N \rightarrow \infty} \sum_{P=1}^{N} \mathbf{u}\left(x_{P}, y_{P}, z_{P}\right) \cdot \mathbf{n}_{P} \Delta S_{P}^{(\beta)},
\end{aligned}
$$

where for $P=1,2, \ldots, N$ and $N$ elements of area $\Delta S_{P}^{(\beta)}$ with a unit normal local fractional vector $n_{P}$, it is required that all $\Delta S_{P}^{(\beta)} \rightarrow 0$ as $N \rightarrow \infty$.

The local fractional volume integral of the given function (9) in a fractal region $V^{(\gamma)}$ is given by [27]

$$
\iiint_{V^{(\gamma)}} \mathbf{u}\left(x_{P}, y_{P}, z_{P}\right) d V^{(\gamma)}=\lim _{N \rightarrow \infty} \sum_{P=1}^{N} \mathbf{u}\left(x_{P}, y_{P}, z_{P}\right) \Delta V_{P}^{(\gamma)},
$$

where for $P=1,2, \ldots, N$ and $N$ elements of volume $\Delta V_{P}^{(\gamma)}$, it is required that all $\Delta V_{P}^{(\gamma)} \rightarrow 0$ as $N \rightarrow \infty$.

Let us consider a local fractional vector field $\mathbf{u}=u_{1} \mathbf{e}_{1}^{\alpha}+$ $u_{2} \mathbf{e}_{2}^{\alpha}+u_{3} \mathbf{e}_{3}^{\alpha}$, the following results hold [27]:

(a) Divergence Theorem of local fractional field states that

$$
\iiint_{V^{(\gamma)}} \nabla^{\alpha} \cdot \mathbf{u} d V^{(\gamma)}=\oiint_{S^{(\beta)}} \mathbf{u} \cdot d \mathbf{S}^{(\beta)} .
$$

(b) Stokes' theorem of local fractional field states that

$$
\oint_{l^{(\alpha)}} \mathbf{u} \cdot d \mathbf{l}^{\alpha}=\iint_{S^{(\beta)}}\left(\nabla^{\alpha} \times \mathbf{u}\right) \cdot d \mathbf{S}^{(\beta)} .
$$

(c) Green's first theorem in fractal domain states that

$$
\begin{aligned}
& \oiint_{S^{(\beta)}} \phi \nabla^{\alpha} \varphi \cdot d \mathbf{S}^{(\beta)} \\
& =\iiint_{V^{(\gamma)}}\left(\phi \nabla^{2 \alpha} \varphi+\left(\nabla^{\alpha} \phi\right) \cdot\left(\nabla^{\alpha} \varphi\right)\right) d V^{(\gamma)} .
\end{aligned}
$$


(d) Green's second theorem in fractal domain states that

$$
\begin{aligned}
& \oiint_{S^{(\beta)}}\left(\phi \nabla^{\alpha} \varphi-\phi \nabla^{\alpha} \varphi\right) \cdot d \mathbf{S}^{(\beta)} \\
& =\iiint_{V^{(\gamma)}}\left(\phi \nabla^{2 \alpha} \varphi-\varphi \nabla^{2 \alpha} \phi\right) d V^{(\gamma)},
\end{aligned}
$$

where the relationships of fractal dimensions of line, surface, and volume fields are

$$
\beta=2 \alpha, \quad \gamma=3 \alpha .
$$

\section{Fractal Kinematics, Continuity Equations, and Constitutive Equations for Newtonian Fluid on Cantor Sets}

In this section, we investigate the fractal kinematics, balance of mass on Cantor sets, and constitutive equations for Newtonian fluid on Cantor sets. We first start with fractal kinematics.

3.1. Fractal Kinematics. For any scalar property $\psi$ associated with an abstract Cantor body B expressed as

$$
\psi=\psi(P, t)=\widehat{\psi}(X, t)=\widetilde{\psi}(x, t),
$$

we define the following Lagrangian and Eulerian temporal and spatial derivatives with local fractional differential operator:

$$
\begin{gathered}
\dot{\psi}=\frac{D^{\alpha} \widehat{\psi}}{D t^{\alpha}}, \quad \psi_{, t}^{\alpha}=\frac{\partial^{\alpha} \tilde{\psi}}{\partial t^{\alpha}}, \quad \nabla_{X}^{\alpha} \psi=\frac{\partial^{\alpha} \widehat{\psi}}{\partial X^{\alpha}}, \\
\nabla_{x}^{\alpha} \psi=\nabla^{\alpha} \psi=\frac{\partial^{\alpha} \tilde{\psi}}{\partial x^{\alpha}} .
\end{gathered}
$$

The velocity $v$ and the acceleration a with local fractional operator are defined through

$$
\boldsymbol{v}=\frac{D^{\alpha} \chi}{D t^{\alpha}}, \quad \mathbf{a}=\frac{D^{2 \alpha} \chi}{D t^{2 \alpha}}
$$

It immediately follows that the velocity gradient $\Theta$ and its symmetric part $\Lambda$ are expressed through

$$
\Theta=\nabla^{\alpha} \cdot \boldsymbol{v}, \quad \Theta^{T}=\boldsymbol{v} \cdot \nabla^{\alpha}, \quad \boldsymbol{\Lambda}=\frac{1}{2}\left(\Theta+\Theta^{T}\right) .
$$

The deformation gradient $\Sigma$ is given by

$$
\Sigma=\nabla^{\alpha} \cdot \chi
$$

which leads to

$$
\boldsymbol{\Theta}=\frac{\partial^{\alpha} \Sigma}{\partial t^{\alpha}} \Sigma^{-1}
$$

The fractal material derivative of the fluid density $\rho$ is defined as [27]

$$
\frac{D^{\alpha} \rho}{D t^{\alpha}}=\frac{\partial^{\alpha} \rho}{\partial t^{\alpha}}+v \cdot \nabla^{\alpha} \rho .
$$

3.2. Balance of Mass on Cantor Sets. The mass of fractal fluid in $V^{(\gamma)}$ is defined through [27]

$$
M=\iiint_{V^{(\gamma)}} \rho d V^{(\gamma)},
$$

which yields that the balance of mass on Cantor sets takes the form [27]

$$
\frac{\partial^{\alpha} M}{\partial t^{\alpha}}=-\oiint_{S^{(\beta)}} \rho \boldsymbol{v} \cdot d \mathbf{S}^{(\beta)} .
$$

Using Divergence Theorem of local fractional field, we deduce to

$$
\begin{aligned}
& \iiint_{V^{(\gamma)}} \frac{\partial^{\alpha}}{\partial t^{\alpha}} \rho d V^{(\gamma)}+\oiint_{S^{(\beta)}} \rho \boldsymbol{v} \cdot d \mathbf{S}^{(\beta)} \\
& \quad=\iiint_{V^{(\gamma)}}\left[\frac{\partial^{\alpha} \rho}{\partial t^{\alpha}}+\nabla^{\alpha} \cdot(\rho \boldsymbol{v})\right] d V^{(\gamma)}=0
\end{aligned}
$$

which implies that

$$
\frac{\partial^{\alpha} \rho}{\partial t^{\alpha}}+\nabla^{\alpha} \cdot(\rho \boldsymbol{v})=0
$$

This is called continuity equation on Cantor sets.

It becomes

$$
\begin{aligned}
\frac{\partial^{\alpha} \rho}{\partial t^{\alpha}}+\nabla^{\alpha} \cdot(\rho \boldsymbol{v}) & =\frac{\partial^{\alpha} \rho}{\partial t^{\alpha}}+\boldsymbol{v} \cdot\left(\nabla^{\alpha} \rho\right)+\rho\left(\nabla^{\alpha} \cdot \boldsymbol{v}\right) \\
& =\frac{D^{\alpha} \rho}{D t^{\alpha}}+\rho\left(\nabla^{\alpha} \cdot \boldsymbol{v}\right)=0,
\end{aligned}
$$

where the fractal material derivative of the fluid density $\rho$ is noted by

$$
\frac{D^{\alpha} \rho}{D t^{\alpha}}=\frac{\partial^{\alpha} \rho}{\partial t^{\alpha}}+v \cdot\left(\nabla^{\alpha} \rho\right) .
$$

If the fractal fluid is incompressible, we deduce that

$$
\frac{\partial^{\alpha} \rho}{\partial t^{\alpha}}+\boldsymbol{v} \cdot\left(\nabla^{\alpha} \rho\right)=0 \Longleftrightarrow \frac{D^{\alpha} \rho}{D t^{\alpha}}=0 \quad \text { or } \quad \nabla^{\alpha} \cdot \boldsymbol{v}=0 .
$$

It follows that Reynolds transport theorem on Cantor sets states that

$$
\begin{aligned}
\frac{D^{\alpha}}{D t^{\alpha}} \iiint_{V^{(\gamma)}} F(x, t) d V^{(\gamma)}= & \iiint_{V^{(\gamma)}} \frac{\partial^{\alpha}}{\partial t^{\alpha}} F(x, t) d V^{(\gamma)} \\
& +\oiint_{S^{(\beta)}} F(x, t) v \cdot d \mathbf{S}^{(\beta)},
\end{aligned}
$$

where $v$ is the fractal fluid velocity, $V^{(\gamma)}$ is the fractal material volume, and $\mathbf{S}^{(\beta)}$ is the fractal surfaces moving with the fractal fluid.

3.3. Constitutive Equations for Newtonian Fluid on Cantor Sets. We will assume a linear relation of the type of fractal Cauchy stress

$$
\mathbf{J}=-p \mathbf{I}+\mathbf{K}: \Lambda,
$$


where $p$ is the thermodynamic pressure, $\mathbf{K}$ is a fourth-order fractal tensor, $\boldsymbol{\Lambda}$ is the fractal strain rate tensor, and $\mathbf{I}$ is unit vector in local fractional field.

The fractal velocity gradient tensor can be decomposed into symmetric and antisymmetric parts [27]

$$
\nabla^{\alpha} \cdot \boldsymbol{v}=\frac{1}{2}\left(\Theta+\Theta^{T}\right)+\frac{1}{2}\left(\Theta-\Theta^{T}\right)=\Lambda+\frac{1}{2}\left(\Theta-\Theta^{T}\right) .
$$

We can write

$$
\mathbf{K}: \boldsymbol{\Lambda}=2 \mu \boldsymbol{\Lambda}+\lambda\left(\nabla^{\alpha} \cdot \boldsymbol{v}\right) \mathbf{I}
$$

which leads to

$$
\mathbf{J}=-p \mathbf{I}+2 \mu \boldsymbol{\Lambda}+\lambda\left(\nabla^{\alpha} \cdot \boldsymbol{v}\right) \mathbf{I}
$$

where $\left(\nabla^{\alpha} \cdot v\right)$ is the fractal volumetric strain rate and $\boldsymbol{\Lambda}=$ $(1 / 2)\left(\nabla^{\alpha} \cdot \boldsymbol{v}+\boldsymbol{v} \cdot \nabla^{\alpha}\right)$ [27], $\lambda$ and $\mu$ are the bulk and shear moduli of viscosity.

The constitutive equation of homogeneous compressible Euler fluid on Cantor sets reads

$$
\mathbf{J}=-p(\rho) \mathbf{I}
$$

The constitutive equation of homogeneous incompressible Euler fluid on Cantor sets is

$$
\mathbf{J}=-p \mathbf{I}, \quad \nabla^{\alpha} \cdot \boldsymbol{v}=0 .
$$

The constitutive equation of homogeneous compressible Navier-Stokes fluid on Cantor sets is written in the form

$$
\mathbf{J}=-p(\rho) \mathbf{I}+2 \mu(\rho) \boldsymbol{\Lambda}+\lambda(\rho)\left(\nabla^{\alpha} \cdot \boldsymbol{v}\right) I .
$$

The constitutive equation of homogeneous incompressible Navier-Stokes fluid on Cantor sets is expressed as

$$
\mathbf{J}=-p \mathbf{I}+2 \mu \boldsymbol{\Lambda}, \quad \nabla^{\alpha} \cdot \boldsymbol{v}=0 .
$$

By using Stokes' assumption $\lambda(\rho)=-2 \mu(\rho) / 3$, the constitutive equation of homogeneous compressible Navier-Stokes fluid on Cantor sets becomes

$$
\mathbf{J}=-p(\rho) \mathbf{I}+2 \mu(\rho)\left(\nabla^{\alpha} \cdot \boldsymbol{v}+\boldsymbol{v} \cdot \nabla^{\alpha}\right)-\frac{2}{3} \mu(\rho)\left(\nabla^{\alpha} \cdot \boldsymbol{v}\right),
$$

while the constitutive equation of homogeneous incompressible Navier-Stokes fluid on Cantor sets can obtain that

$$
\mathbf{J}=-p \mathbf{I}+\mu\left(\nabla^{\alpha} \cdot \boldsymbol{v}+\boldsymbol{v} \cdot \nabla^{\alpha}\right), \quad \nabla^{\alpha} \cdot \boldsymbol{v}=0 .
$$

\section{Cauchy's Equations of Motion and Mechanical Energy Equations on Cantor Sets}

In this section, we consider balance of linear and angular momentums and energy on Cantor sets.
4.1. Balances of Linear and Angular Momentums on Cantor Sets. If the second law of Newton in fractal mechanics is valid, the balance of linear momentum for flows on Cantor sets takes the form [27]

$$
\frac{D^{\alpha}}{D t^{\alpha}} \iiint_{V^{(\gamma)}} \rho \boldsymbol{v} d V^{(\gamma)}=\iiint_{V^{(\gamma)}} \rho \mathbf{b} d V^{(\gamma)}+\oiint_{S^{(\beta)}} \mathbf{J} \cdot d \mathbf{S}^{(\beta)},
$$

where J denotes the fractal Cauchy stress tensor [27] and b denotes the specific fractal body force [27].

By using (32), (43) becomes

$$
\begin{aligned}
\frac{D^{\alpha}}{D t^{\alpha}} \iiint_{V^{(\gamma)}} \rho \boldsymbol{v} d V^{(\gamma)}= & \iiint_{V^{(\gamma)}} \frac{\partial^{\alpha}}{\partial t^{\alpha}}(\rho \boldsymbol{v}) d V^{(\gamma)} \\
& +\oiint_{S^{(\beta)}} \rho \boldsymbol{v} \boldsymbol{v} \cdot d \mathbf{S}^{(\beta)} \\
= & \iiint_{V^{(\gamma)}} \rho \mathbf{b} d V^{(\gamma)}+\oiint_{S^{(\beta)}} \mathbf{J} \cdot d \mathbf{S}^{(\beta)}
\end{aligned}
$$

which is rewritten as

$$
\frac{\partial^{\alpha}}{\partial t^{\alpha}}(\rho \boldsymbol{v})+\nabla^{\alpha} \cdot[\rho \boldsymbol{v} \boldsymbol{v}]=\rho \mathbf{b}+\nabla^{\alpha} \cdot \mathbf{J} .
$$

In view of (7), (45) yields

$$
\frac{\partial^{\alpha} \rho}{\partial t^{\alpha}} \boldsymbol{v}+\rho \frac{\partial^{\alpha} \boldsymbol{v}}{\partial t^{\alpha}}+\boldsymbol{v} \boldsymbol{v} \cdot \nabla^{\alpha} \rho+\rho \boldsymbol{v} \nabla^{\alpha} \cdot \boldsymbol{v}+\rho \boldsymbol{v} \cdot \nabla^{\alpha} \boldsymbol{v}=\rho \mathbf{b}+\nabla^{\alpha} \cdot \mathbf{J}
$$

which implies that

$$
\boldsymbol{v}\left(\frac{\partial^{\alpha} \rho}{\partial t^{\alpha}}+\nabla^{\alpha}(\boldsymbol{v} \cdot \rho)\right)+\rho\left(\frac{\partial^{\alpha} \boldsymbol{v}}{\partial t^{\alpha}}+\boldsymbol{v} \cdot \nabla^{\alpha} \boldsymbol{v}\right)=\rho \mathbf{b}+\nabla^{\alpha} \cdot \mathbf{J}
$$

or

$$
\begin{aligned}
& \boldsymbol{v}\left(\frac{\partial^{\alpha} \rho}{\partial t^{\alpha}}+\boldsymbol{v} \cdot \nabla^{\alpha} \rho+\rho \nabla^{\alpha} \cdot \boldsymbol{v}\right)+\rho\left(\frac{\partial^{\alpha} \boldsymbol{v}}{\partial t^{\alpha}}+\boldsymbol{v} \cdot \nabla^{\alpha} \boldsymbol{v}\right) \\
& \quad=\rho \mathbf{b}+\nabla^{\alpha} \cdot \mathbf{J} .
\end{aligned}
$$

Therefore, (48) is reexpressed by

$$
\boldsymbol{v}\left(\frac{\partial^{\alpha} \rho}{\partial t^{\alpha}}+\nabla^{\alpha} \cdot(\rho \boldsymbol{v})\right)+\rho\left(\frac{\partial^{\alpha} \boldsymbol{v}}{\partial t^{\alpha}}+\boldsymbol{v} \cdot \nabla^{\alpha} \boldsymbol{v}\right)=\rho \mathbf{b}+\nabla^{\alpha} \cdot \mathbf{J}
$$

Taking continuity equation on Cantor sets and fractal material derivative yields

$$
\rho \frac{D^{\alpha} \boldsymbol{v}}{D t^{\alpha}}=\rho \mathbf{b}+\nabla^{\alpha} \cdot \mathbf{J} .
$$

Hence, balance of linear momentum in its local Cantorian form (the Newton's law in its local Cantorian form) is rewritten as

$$
\rho \frac{D^{\alpha} \boldsymbol{v}}{D t^{\alpha}}=\nabla^{\alpha} \cdot \mathbf{J}+\rho \mathbf{b}
$$


or

$$
\rho \frac{\partial^{\alpha} \boldsymbol{v}}{\partial t^{\alpha}}=\nabla^{\alpha} \cdot \mathbf{J}+\rho \mathbf{b}-\rho \boldsymbol{v}\left(\nabla^{\alpha} \cdot \boldsymbol{v}\right)
$$

It is called as the Cauchy's equation of motion of flows on Cantor sets.

In the absence of internal couples, the balance of angular momentum on Cantor sets implies that the fractal Cauchy stress is symmetric [27]; that is,

$$
\rho \frac{D^{\alpha} \boldsymbol{v}}{D t^{\alpha}}=\nabla^{\alpha} \cdot \mathbf{J}^{T}+\rho \mathbf{b} \quad \text { or } \quad \mathbf{J}=\mathbf{J}^{T} .
$$

Taking fractal material derivative, (45) is expressed by

$$
\rho \frac{\partial^{\alpha} \boldsymbol{v}}{\partial t^{\alpha}}+\rho \boldsymbol{v}\left(\nabla^{\alpha} \cdot \boldsymbol{v}\right)=\nabla^{\alpha} \cdot \mathbf{J}^{T}+\rho \mathbf{b}
$$

or

$$
\mathbf{J}=\mathbf{J}^{T}
$$

which yields the Newton's law in local fractional integration form given by

$$
\iiint_{V^{(\gamma)}} \nabla^{\alpha} \cdot\left(\rho \boldsymbol{v} \cdot \boldsymbol{v}-\mathbf{J}^{T}\right) d V^{(\gamma)}=\oiint_{S^{(\beta)}}\left[\rho \boldsymbol{v} \cdot \boldsymbol{v}-\mathbf{J}^{T}\right] d \mathbf{S}^{(\beta)}
$$

if the compressible flow on Cantor sets is steady and body forces are absent. The results are different from [36-38] because of the fractional operators.

In local Eulerian form, Cauchy's equation of motion of flows on Cantor sets is given by using (37)

$$
\frac{D^{\alpha}}{D t^{\alpha}} \iiint_{V^{(\gamma)}} \rho \boldsymbol{v} d V^{(\gamma)}=\iiint_{V^{(\gamma)}} \rho \mathbf{b} d V^{(\gamma)}-\oiint_{S^{(\beta)}} p \mathbf{I} \cdot d \mathbf{S}^{(\beta)}
$$

which implies that

$$
\rho \frac{D^{\alpha} \boldsymbol{v}}{D t^{\alpha}}=-\nabla^{\alpha} p(\rho)+\rho \mathbf{b}
$$

For compressible fluid, Cauchy's equation of motion of flows on Cantor sets is obtained by using (39)

$$
\begin{aligned}
& \frac{D^{\alpha}}{D t^{\alpha}} \iiint_{V^{(\gamma)}} \rho \boldsymbol{v} d V^{(\gamma)} \\
& =\iiint_{V^{(\gamma)}} \rho \mathbf{b} d V^{(\gamma)} \\
& \quad-\oiint_{S^{(\beta)}}\left(p(\rho) \mathbf{I}+\mu(\rho)\left(\frac{1}{3}\left(\nabla^{\alpha} \cdot \boldsymbol{v}\right)+\boldsymbol{v} \cdot \nabla^{\alpha}\right)\right) \cdot d \mathbf{S}^{(\beta)}
\end{aligned}
$$

which is

$$
\begin{aligned}
\rho \frac{D^{\alpha} \boldsymbol{v}}{D t^{\alpha}}= & -\nabla^{\alpha} p(\rho)+\frac{1}{3} \nabla^{\alpha} \cdot\left[\mu(\rho)\left(\nabla^{\alpha} \cdot \boldsymbol{v}\right)\right] \\
& +\nabla^{\alpha} \cdot\left(\mu(\rho) \boldsymbol{v} \cdot \nabla^{\alpha}\right)+\rho \mathbf{b} .
\end{aligned}
$$

For incompressible fluid, Cauchy's equation of motion of flows on Cantor sets is obtained by using (40)

$$
\begin{aligned}
& \frac{D^{\alpha}}{D t^{\alpha}} \iiint_{V^{(\gamma)}} \rho \boldsymbol{v} d V^{(\gamma)} \\
& \quad=\iiint_{V^{(\gamma)}} \rho \mathbf{b} d V^{(\gamma)} \\
& \quad+\oiint_{S^{(\beta)}}\left(-p \mathbf{I}+\mu\left(\nabla^{\alpha} \cdot \boldsymbol{v}+\boldsymbol{v} \cdot \nabla^{\alpha}\right)\right) \cdot d \mathbf{S}^{(\beta)}
\end{aligned}
$$

which leads to

$$
\rho \frac{D^{\alpha} \boldsymbol{v}}{D t^{\alpha}}=-\nabla^{\alpha} p+\mu \nabla^{\alpha}\left(\boldsymbol{v} \cdot \nabla^{\alpha}\right)+\rho \mathbf{b}
$$

or

$$
\rho \frac{D^{\alpha} v}{D t^{\alpha}}=-\nabla^{\alpha} p+\mu \nabla^{\alpha}\left(v \cdot \nabla^{\alpha}\right)
$$

4.2. Balance of Energy on Cantor Sets. The mechanical energy equation with viscous dissipation on Cantor sets is repeated by

$$
\begin{aligned}
& \frac{D^{\alpha}}{D t^{\alpha}} \iiint_{V^{(\gamma)}}(\rho \varphi) d V^{(\gamma)}+\oiint_{S^{(\beta)}}(\rho \varphi \boldsymbol{v}) \cdot d \mathbf{S}^{(\beta)} \\
& =\iiint_{V^{(\gamma)}}(\rho \mathbf{b} \cdot \boldsymbol{v}) d V^{(\gamma)}+\oiint_{S^{(\beta)}} \boldsymbol{v} \cdot \mathbf{J} d \mathbf{S}^{(\beta)} \\
& \quad-\iiint_{V^{(\gamma)}} \nabla^{\alpha} \cdot(p \boldsymbol{v}) d V^{(\gamma)}-\iiint_{V^{(\gamma)}} \phi d V^{(\gamma)},
\end{aligned}
$$

where the first term is rate of change of kinetic energy in fractal domain, the second term is rate of outflow across fractal boundary, the third term is rate of work by fractal body force, the fourth term is rate of work by surface force, the fifth term is rate of work by volume expansion, and the last term is rate of viscous dissipation.

Local fractional differential form of the mechanical energy equation with viscous dissipation on Cantor sets gives

$$
\begin{aligned}
\frac{D^{\alpha}}{D t^{\alpha}}(\rho \varphi)+\nabla^{\alpha} \cdot(\rho \varphi \boldsymbol{v})= & \rho \mathbf{b} \cdot \boldsymbol{v}+\boldsymbol{v} \cdot\left(\nabla^{\alpha} \cdot \mathbf{J}\right) \\
& +\nabla^{\alpha} \cdot(p \boldsymbol{v})-\phi
\end{aligned}
$$

which implies for fractal material derivative of $\rho \varphi$

$$
\begin{aligned}
& \frac{\partial^{\alpha}}{\partial t^{\alpha}}(\rho \varphi)+\boldsymbol{v} \cdot \nabla^{\alpha}(\rho \varphi)+\nabla^{\alpha} \cdot(\rho \varphi \boldsymbol{v}) \\
& \quad=\rho \mathbf{b} \cdot \boldsymbol{v}+\boldsymbol{v} \cdot\left(\nabla^{\alpha} \cdot \mathbf{J}\right)-\nabla^{\alpha} \cdot(p \boldsymbol{v})-\phi,
\end{aligned}
$$

where the rate of viscous dissipation of kinetic energy per unit volume is $\phi$. 
The integration form of the balance of energy on Cantor sets is expressed by

$$
\begin{aligned}
& \frac{D^{\alpha}}{D t^{\alpha}} \iiint_{V^{(\gamma)}} \rho(\theta+\varphi) d V^{(\gamma)} \\
& =\oiint_{S^{(\beta)}} \boldsymbol{v} \cdot \mathbf{J} \cdot d \mathbf{S}^{(\beta)}-\oiint_{S^{(\beta)}} \rho(\theta+\varphi) \boldsymbol{v} \cdot d \mathbf{S}^{(\beta)} \\
& \quad+\iiint_{V^{(\gamma)}}(\rho \mathbf{b} \cdot \boldsymbol{v}) d V^{(\gamma)}+\oiint_{S^{(\beta)}} K^{2 \alpha} \mathbf{q} \cdot d \mathbf{S}^{(\beta)} \\
& \quad-\iiint_{V^{(\gamma)}} \phi d V^{(\gamma)}
\end{aligned}
$$

which leads to

$$
\begin{aligned}
\frac{D^{\alpha}}{D t^{\alpha}}[\rho(\theta+\varphi)]= & \boldsymbol{v} \cdot\left(\nabla^{\alpha} \cdot \mathbf{J}\right)+\rho \mathbf{b} \cdot \boldsymbol{v}-\nabla^{\alpha} \cdot(p \boldsymbol{v}) \\
& -\nabla^{\alpha} \cdot\left[\rho(\theta+\varphi) \boldsymbol{v}-K^{2 \alpha} \cdot \mathbf{q}\right]-\phi,
\end{aligned}
$$

where $K^{2 \alpha}$ is denoted as fractal heat conduction coefficient and $\mathbf{q}$ is fractal temperature flied.

The term

$$
\oiint_{S^{(\beta)}} \rho(\theta+\varphi) \boldsymbol{v} \cdot d \mathbf{S}^{(\beta)}=\iiint_{V^{(\gamma)}} \nabla^{\alpha} \cdot[\rho(\theta+\varphi)] v d V^{(\gamma)}
$$

vanishes if the term $\rho \theta v$ is zero at the boundaries and

$$
\begin{aligned}
\iiint_{V^{(\gamma)}}\left(p\left(\nabla^{\alpha} \cdot \boldsymbol{v}\right)\right) d V^{(\gamma)}= & \oiint_{S^{(\beta)}}(p \boldsymbol{v}) d \mathbf{S}^{(\beta)} \\
& -\iiint_{V^{(\gamma)}}\left[\boldsymbol{v} \cdot\left(\nabla^{\alpha} p\right)\right] d V^{(\gamma)} .
\end{aligned}
$$

The mechanical energy equation with no viscous dissipation on Cantor sets is written in the local Cantorian form as

$$
\rho \frac{D^{\alpha}}{D t^{\alpha}}(\theta+\varphi)=-\nabla^{\alpha} p \cdot \boldsymbol{v}+\boldsymbol{v} \cdot\left(\nabla^{\alpha} \cdot \mathbf{J}\right)+\rho \mathbf{b} \cdot \boldsymbol{v}+K^{2 \alpha} \nabla^{\alpha} \cdot \mathbf{q}
$$

where $\varphi$ is the kinetic energy per unit of mass, $\mathbf{b}$ is the external force per unit of mass, $\mathbf{J}$ is the fractal Cauchy stress tensor, and $\theta$ is the internal energy per unit of mass.

\section{Systems of Navier-Stokes Equation on Cantor Sets}

In this section, we investigate the Navier-Stokes equation on Cantor sets and derive systems of Navier-Stokes equation on Cantor sets.

5.1. Navier-Stokes Equation on Cantor Sets. Substituting the constitutive equation into Cauchy's equation (41) yields

$$
\begin{aligned}
\rho \frac{D^{\alpha} \boldsymbol{v}}{D t^{\alpha}}= & -\nabla^{\alpha} \cdot(p \mathbf{I}) \\
& +\nabla^{\alpha}\left[2 \mu\left(\nabla^{\alpha} \cdot \boldsymbol{v}+\boldsymbol{v} \cdot \nabla^{\alpha}\right)-\frac{2}{3} \mu\left(\nabla^{\alpha} \cdot \boldsymbol{v}\right) \mathbf{I}\right]+\rho \mathbf{b},
\end{aligned}
$$

where viscosity $\mu$ in this equation can be a function of the thermodynamic state.

This is a general form of the Navier-Stokes equation on Cantor sets, which is the equation of motion for a Newtonian fluid on Cantor sets.

For incompressible fluids $\nabla^{\alpha} \cdot \boldsymbol{v}=0$, we deduce to

$$
\rho \frac{D^{\alpha} \boldsymbol{v}}{D t^{\alpha}}=-\nabla^{\alpha} p+\mu \nabla^{2 \alpha} \boldsymbol{v}+\rho \mathbf{b} .
$$

Applying fractal material derivative of the fluid velocity,

$$
\frac{D^{\alpha} v}{D t^{\alpha}}=\frac{\partial^{\alpha} v}{\partial t^{\alpha}}+v\left(\nabla^{\alpha} \cdot v\right)
$$

in compressible fluids, a general form of the Navier-Stokes equation on Cantor sets is stated as

$$
\rho \frac{\partial^{\alpha} \boldsymbol{v}}{\partial t^{\alpha}}=-\nabla^{\alpha} p+\mu \nabla^{2 \alpha} \boldsymbol{v}+\rho \mathbf{b}-\rho \boldsymbol{v}\left(\nabla^{\alpha} \cdot \boldsymbol{v}\right) .
$$

For compressible fluid, the Navier-Stokes equation on Cantor sets reads

$$
\begin{aligned}
\rho \frac{D^{\alpha} \boldsymbol{v}}{D t^{\alpha}}= & -\nabla^{\alpha} p+\nabla^{\alpha}\left[\mu\left(\nabla^{\alpha} \cdot \boldsymbol{v}+\boldsymbol{v} \cdot \nabla^{\alpha}\right)\right] \\
& +\nabla^{\alpha}\left(\lambda\left(\nabla^{\alpha} \cdot \boldsymbol{v}\right)\right)+\rho \mathbf{b}
\end{aligned}
$$

which becomes for $\lambda=-(2 / 3) \mu$

$$
\rho \frac{D^{\alpha} \boldsymbol{v}}{D t^{\alpha}}=-\nabla^{\alpha} p+\frac{1}{3} \mu \nabla^{\alpha}\left(\left(\nabla^{\alpha} \cdot \boldsymbol{v}\right)\right)+\mu \nabla^{2 \alpha} \boldsymbol{v}+\rho \mathbf{b}
$$

which yields by using (55)

$$
\begin{aligned}
\rho \frac{\partial^{\alpha} \boldsymbol{v}}{\partial t^{\alpha}}= & -\nabla^{\alpha} p+\frac{1}{3} \mu \nabla^{\alpha}\left(\left(\nabla^{\alpha} \cdot \boldsymbol{v}\right)\right) \\
& +\mu \nabla^{2 \alpha} \boldsymbol{v}+\rho \mathbf{b}-\rho \boldsymbol{v}\left(\nabla^{\alpha} \cdot \boldsymbol{v}\right)
\end{aligned}
$$

5.2. Systems of Navier-Stokes Equation on Cantor Sets. In this section, we consider systems of Navier-Stokes fluid on Cantor sets, which states that the systems consists of the continuity equation, the motion equation, and the energy balance equation on Cantor sets.

By using (29), (71), and (77), systems of compressible Navier-Stokes equations on Cantor sets become as follows:

$$
\begin{gathered}
\frac{\partial^{\alpha} \rho}{\partial t^{\alpha}}+\nabla^{\alpha} \cdot(\rho \boldsymbol{v})=0 \\
\rho \frac{D^{\alpha} \boldsymbol{v}}{D t^{\alpha}}=-\nabla^{\alpha} p+\frac{1}{3} \mu \nabla^{\alpha}\left(\left(\nabla^{\alpha} \cdot \boldsymbol{v}\right)\right)+\mu \nabla^{2 \alpha} \boldsymbol{v}+\rho \mathbf{b} \\
\rho \frac{D^{\alpha}}{D t^{\alpha}}(\theta+\varphi)=-\nabla^{\alpha} \cdot(p \boldsymbol{v})+\boldsymbol{v} \cdot\left(\nabla^{\alpha} \cdot \mathbf{J}\right) \\
+\rho \mathbf{b} \cdot \boldsymbol{v}+K^{2 \alpha} \nabla^{\alpha} \cdot \mathbf{q} .
\end{gathered}
$$


By applying (31), (71), and (73), systems of incompressible Navier-Stokes equations on Cantor sets are stated as

$$
\begin{gathered}
\nabla^{\alpha} \cdot \boldsymbol{v}=0, \\
\rho \frac{D^{\alpha} \boldsymbol{v}}{D t^{\alpha}}=-\nabla^{\alpha} p+\mu \nabla^{2 \alpha} \boldsymbol{v}+\rho \mathbf{b}, \\
\rho \frac{D^{\alpha}}{D t^{\alpha}}(\theta+\varphi)=-\nabla^{\alpha} \cdot(p \boldsymbol{v})+\boldsymbol{v} \cdot\left(\nabla^{\alpha} \cdot \mathbf{J}\right) \\
+\rho \mathbf{b} \cdot \boldsymbol{v}+K^{2 \alpha} \nabla^{\alpha} \cdot \mathbf{q},
\end{gathered}
$$

or in the 3D Cantorian coordinates, systems of Navier-Stokes equations on Cantor sets can be written for each component, $x, y$, and $z$ as

$$
\begin{gathered}
\frac{\partial^{\alpha} v_{x}}{\partial x^{\alpha}}+\frac{\partial^{\alpha} v_{y}}{\partial y^{\alpha}}+\frac{\partial^{\alpha} v_{z}}{\partial z^{\alpha}}=0, \\
\rho \frac{D^{\alpha} v_{x}}{D t^{\alpha}}=-\frac{\partial^{\alpha} p}{\partial x^{\alpha}}+\mu\left(\frac{\partial^{2 \alpha} v_{x}}{\partial x^{2 \alpha}}+\frac{\partial^{2 \alpha} v_{x}}{\partial y^{2 \alpha}}+\frac{\partial^{2 \alpha} v_{x}}{\partial z^{2 \alpha}}\right)+\rho b_{x}, \\
\rho \frac{D^{\alpha} v_{y}}{D t^{\alpha}}=-\frac{\partial^{\alpha} p}{\partial y^{\alpha}}+\mu\left(\frac{\partial^{2 \alpha} v_{y}}{\partial x^{2 \alpha}}+\frac{\partial^{2 \alpha} v_{y}}{\partial y^{2 \alpha}}+\frac{\partial^{2 \alpha} v_{y}}{\partial z^{2 \alpha}}\right)+\rho b_{y}, \\
\rho \frac{D^{\alpha} v_{z}}{D t^{\alpha}}=-\frac{\partial^{\alpha} p}{\partial z^{\alpha}}+\mu\left(\frac{\partial^{2 \alpha} v_{z}}{\partial x^{2 \alpha}}+\frac{\partial^{2 \alpha} v_{z}}{\partial y^{2 \alpha}}+\frac{\partial^{2 \alpha} v_{z}}{\partial z^{2 \alpha}}\right)+\rho b_{z}, \\
\rho \frac{D^{\alpha}}{D t^{\alpha}}(\theta+\varphi)=-\left(\frac{\partial^{\alpha}\left(p v_{x}\right)}{\partial x^{\alpha}}+\frac{\partial^{\alpha}\left(p v_{y}\right)}{\partial y^{\alpha}}+\frac{\partial^{\alpha}\left(p v_{z}\right)}{\partial z^{\alpha}}\right) \\
+v_{x}\left(\frac{\partial^{\alpha} J_{x x}}{\partial x^{\alpha}}+\frac{\partial^{\alpha} J_{x y}}{\partial y^{\alpha}}+\frac{\partial^{\alpha} J_{x z}}{\partial z^{\alpha}}\right) \\
+v_{y}\left(\frac{\partial^{\alpha} J_{y x}}{\partial x^{\alpha}}+\frac{\partial^{\alpha} J_{y y}}{\partial y^{\alpha}}+\frac{\partial^{\alpha} J_{y z}}{\partial z^{\alpha}}\right) \\
+v_{z}\left(\frac{\partial^{\alpha} J_{z x}}{\partial x^{\alpha}}+\frac{\partial^{\alpha} J_{z y}}{\partial y^{\alpha}}+\frac{\partial^{\alpha} J_{z z}}{\partial z^{\alpha}}\right) \\
+\rho b_{x} v_{x}+\rho b_{y} v_{y}+\rho b_{z} v_{z} \\
+K^{2 \alpha}\left(\frac{\partial^{\alpha} q_{x}}{\partial x^{\alpha}}+\frac{\partial^{\alpha} q_{y}}{\partial y^{\alpha}}+\frac{\partial^{\alpha} q_{z}}{\partial z^{\alpha}}\right) .
\end{gathered}
$$

In the 2D Cantorian coordinates, systems of incompressible Navier-Stokes equations on Cantor sets are rewritten in the form

$$
\begin{gathered}
\frac{\partial^{\alpha} v_{x}}{\partial x^{\alpha}}+\frac{\partial^{\alpha} v_{y}}{\partial y^{\alpha}}=0, \\
\rho \frac{D^{\alpha} v_{x}}{D t^{\alpha}}=-\frac{\partial^{\alpha} p}{\partial x^{\alpha}}+\mu\left(\frac{\partial^{2 \alpha} v_{x}}{\partial x^{2 \alpha}}+\frac{\partial^{2 \alpha} v_{x}}{\partial y^{2 \alpha}}\right)+\rho b_{x}, \\
\rho \frac{D^{\alpha} v_{y}}{D t^{\alpha}}=-\frac{\partial^{\alpha} p}{\partial y^{\alpha}}+\mu\left(\frac{\partial^{2 \alpha} v_{y}}{\partial x^{2 \alpha}}+\frac{\partial^{2 \alpha} v_{y}}{\partial y^{2 \alpha}}\right)+\rho b_{y},
\end{gathered}
$$

$$
\begin{aligned}
\rho \frac{D^{\alpha}}{D t^{\alpha}}(\theta+\varphi)= & -\left(\frac{\partial^{\alpha} p v_{x}}{\partial x^{\alpha}}+\frac{\partial^{\alpha} p v_{y}}{\partial y^{\alpha}}\right) \\
& +v_{x}\left(\frac{\partial^{\alpha} J_{x x}}{\partial x^{\alpha}}+\frac{\partial^{\alpha} J_{x y}}{\partial y^{\alpha}}\right) \\
& +v_{y}\left(\frac{\partial^{\alpha} J_{y x}}{\partial x^{\alpha}}+\frac{\partial^{\alpha} J_{y y}}{\partial y^{\alpha}}\right)+\rho b_{x} v_{x}+\rho b_{y} v_{y} \\
& +K^{2 \alpha}\left(\frac{\partial^{\alpha} q_{x}}{\partial x^{\alpha}}+\frac{\partial^{\alpha} q_{y}}{\partial y^{\alpha}}\right) .
\end{aligned}
$$

In the 1D Cantorian coordinates, systems of incompressible Navier-Stokes equations on Cantor sets are repeated by the expression

$$
\begin{gathered}
\frac{\partial^{\alpha} v}{\partial x^{\alpha}}=0, \\
\rho \frac{D^{\alpha} v}{D t^{\alpha}}=-\frac{\partial^{\alpha} p}{\partial x^{\alpha}}+\mu \frac{\partial^{2 \alpha} v}{\partial x^{2 \alpha}}+\rho b, \\
\rho \frac{D^{\alpha}}{D t^{\alpha}}(\theta+\varphi)=-\frac{\partial^{\alpha}(p v)}{\partial x^{\alpha}}+v \frac{\partial^{\alpha} J}{\partial x^{\alpha}}+\rho b v+K^{2 \alpha} \frac{\partial^{\alpha} q}{\partial x^{\alpha}} .
\end{gathered}
$$

\section{Conclusions}

In the present work, we propose the systems of Navier-Stokes equations derived from local fractional vector calculus. These obtained Navier-Stokes equations in one-, two-, and threedimension Cantorian coordinates are shown to describe the materials as being local fractional continuous and nondifferential functions, which are applied to describe fluid flow in fractal media. Comparing between the fractional result in Navier-Stokes equation in fractal media [39] and local fractional one, the former via fractional calculus is continuous and differential quantities as classical result, however, the latter is local fractional continuous and nondifferential quantities. The classical result is obtained in case of fractal space-time dimension, which is equal to 1 .

\section{References}

[1] C. Foias, O. Manley, R. Rosa, and R. Temam, Navier-Stokes Equations and Turbulence, vol. 83, Cambridge University Press, Cambridge, UK, 2001.

[2] G. P. Galdi, An Introduction to the Mathematical Theory of the Navier-Stokes Equations, vol. 1-2, Springer, New York, NY, USA, 1994.

[3] C. Y. Wang, "Exact solutions of the steady-state Navier-Stokes equations," in Annual Review of Fluid Mechanics, vol. 23, pp. 159-177, Annual Reviews, Palo Alto, CA, USA, 1991.

[4] B. Dubrulle, J.-P. Laval, S. Nazarenko, and O. Zaboronski, "A model for rapid stochastic distortions of small-scale turbulence," Journal of Fluid Mechanics, vol. 520, pp. 1-21, 2004.

[5] A. Alexakis, P. D. Mininni, and A. Pouquet, "Imprint of largescale flows on turbulence," Physical Review Letters, vol. 95, no. 26, Article ID 264503, 2005. 
[6] P. D. Mininni, A. Alexakis, and A. Pouquet, "Large-scale flow effects, energy transfer, and self-similarity on turbulence," Physical Review E, vol. 74, no. 1, Article ID 016303, 2006.

[7] R. Benzi, L. Biferale, S. Ciliberto, M. V. Struglia, and R. Tripiccione, "Scaling property of turbulent flows," Physical Review E, vol. 53, no. 4, pp. R3025-R3027, 1996.

[8] Z.-S. She and E. Leveque, "Universal scaling laws in fully developed turbulence," Physical Review Letters, vol. 72, no. 3, pp. 336-339, 1994.

[9] B. I. Shraiman and E. D. Siggia, "Scalar turbulence," Nature, vol. 405, no. 6787, pp. 639-646, 2000.

[10] R. Benzi, S. Ciliberto, R. Tripiccione, C. Baudet, F. Massaioli, and S. Succi, "Extended self-similarity in turbulent flows," Physical Review E, vol. 48, no. 1, pp. R29-R32, 1993.

[11] C. Meneveau and J. Katz, "Scale-invariance and turbulence models for large-eddy simulation," in Annual Review of Fluid Mechanics, vol. 32, pp. 1-32, Annual Reviews, Palo Alto, CA, USA, 2000.

[12] P. Constantin, C. Foias, O. P. Manley, and R. Temam, "Determining modes and fractal dimension of turbulent flows," Journal of Fluid Mechanics, vol. 150, pp. 427-440, 1985.

[13] V. V. Chepyzhov and A. A. Ilyin, "On the fractal dimension of invariant sets: applications to Navier-Stokes equations," Discrete and Continuous Dynamical Systems A, vol. 10, no. 1-2, pp. 117135, 2004.

[14] V. Scheffer, "Turbulence and Hausdorff dimension, in Turbulence and the Navier-Stokes equations," in Lecture Notes in Mathematics, vol. 565, pp. 94-112, Springer, Berlin, Germany, 1976.

[15] V. Scheffer, "Hausdorff measure and the Navier-Stokes equations," Communications in Mathematical Physics, vol. 55, no. 2, pp. 97-112, 1977.

[16] G. Boffetta, A. Mazzino, and A. Vulpiani, “Twenty-five years of multifractals in fully developed turbulence: a tribute to Giovanni Paladin," Journal of Physics A, vol. 41, 363001, 2008.

[17] A. Cheskidov, D. D. Holm, E. Olson, and E. S. Titi, "On a Leray- $\alpha$ model of turbulence," Proceedings of The Royal Society of London A, vol. 461, no. 2055, pp. 629-649, 2005.

[18] A. A. Ilyin, E. M. Lunasin, and E. S. Titi, "A modified-Leray- $\alpha$ subgrid scale model of turbulence," Nonlinearity, vol. 19, no. 4, pp. 879-897, 2006.

[19] D. D. Holm, "Kármán-Howarth theorem for the Lagrangianaveraged Navier-Stokes-alpha model of turbulence," Journal of Fluid Mechanics, vol. 467, pp. 205-214, 2002.

[20] R. Hilfer, Applications of fractional calculus in physics, World Scientific Publishing, River Edge, NJ, USA, 2000.

[21] J. Sabatier, O. P. Agrawal, and J. A. T. Machado, Advances in Fractional Calculus: Theoretical Developments and Applications in Physics and Engineering, Springer, New York, NY, USA, 2007.

[22] A. A. Kilbas, H. M. Srivastava, and J. J. Trujillo, Theory and Applications of Fractional Differential Equations, vol. 204, Elsevier Science, Amsterdam, The Netherlands, 2006.

[23] I. Podlubny, Fractional Differential Equations, vol. 198, Academic Press, New York, NY, USA, 1999.

[24] M. El-Shahed and A. Salem, "On the generalized Navier-Stokes equations," Applied Mathematics and Computation, vol. 156, no. 1, pp. 287-293, 2004.

[25] S. Momani and Z. Odibat, "Analytical solution of a timefractional Navier-Stokes equation by Adomian decomposition method," Applied Mathematics and Computation, vol. 177, no. 2, pp. 488-494, 2006.
[26] Z. Z. Ganji, D. D. Ganji, A. D. Ganji, and M. Rostamian, "Analytical solution of time-fractional Navier-Stokes equation in polar coordinate by homotopy perturbation method," Numerical Methods for Partial Differential Equations, vol. 26, no. 1, pp. 117-124, 2010.

[27] X. J. Yang, Advanced Local Fractional Calculus and Its Applications, World Science Publisher, New York, NY, USA, 2012.

[28] X. J. Yang, D. Baleanu, and J. A. T. Machado, "Mathematical aspects of Heisenberg uncertainty principle within local fractional Fourier analysis," Boundary Value Problems, no. 1, pp. 131150, 2013.

[29] X. J. Yang and D. Baleanu, "Fractal heat conduction problem solved by local fractional variation iteration method," Thermal Science, vol. 17, no. 2, pp. 625-628, 2013.

[30] M.-S. Hu, R. P. Agarwal, and X.-J. Yang, "Local fractional Fourier series with application to wave equation in fractal vibrating string," Abstract and Applied Analysis, vol. 2012, Article ID 567401, 15 pages, 2012.

[31] X. J. Yang, D. Baleanu, and W. P. Zhong, "Approximation solution to diffusion equation on Cantor time-space," Proceedings of the Romanian Academy A, vol. 14, no. 2, pp. 127-133, 2013.

[32] X. J. Yang, Local Fractional Functional Analysis and Its Applications, Asian Academic publisher Limited, Hong Kong, China, 2011.

[33] X. J. Yang, "Local fractional integral transforms," Progress in Nonlinear Science, vol. 4, pp. 1-225, 2011.

[34] A. Carpinteri and A. Sapora, "Diffusion problems in fractal media defined on Cantor sets," ZAMM Zeitschrift fur Angewandte Mathematik und Mechanik, vol. 90, no. 3, pp. 203-210, 2010.

[35] K. M. Kolwankar and A. D. Gangal, "Local fractional FokkerPlanck equation," Physical Review Letters, vol. 80, no. 2, pp. 214217, 1998.

[36] J. Li and M. Ostoja-Starzewski, "Micropolar continuum mechanics of fractal media," International Journal of Engineering Science, vol. 49, no. 12, pp. 1302-1310, 2011.

[37] V. E. Tarasov, "Continuous medium model for fractal media," Physics Letters, Section A, vol. 336, no. 2-3, pp. 167-174, 2005.

[38] C. S. Drapaca and S. Sivaloganathan, "A fractional model of continuum mechanics," Journal of Elasticity, vol. 107, no. 2, pp. 105-123, 2012.

[39] V. E. Tarasov, "Fractional hydrodynamic equations for fractal media," Annals of Physics, vol. 318, no. 2, pp. 286-307, 2005. 


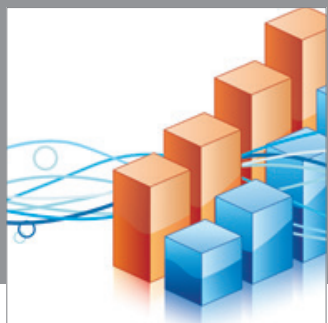

Advances in

Operations Research

mansans

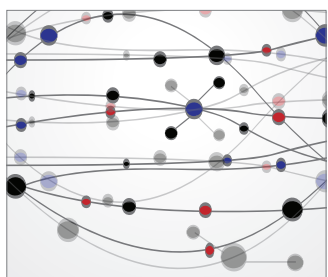

The Scientific World Journal
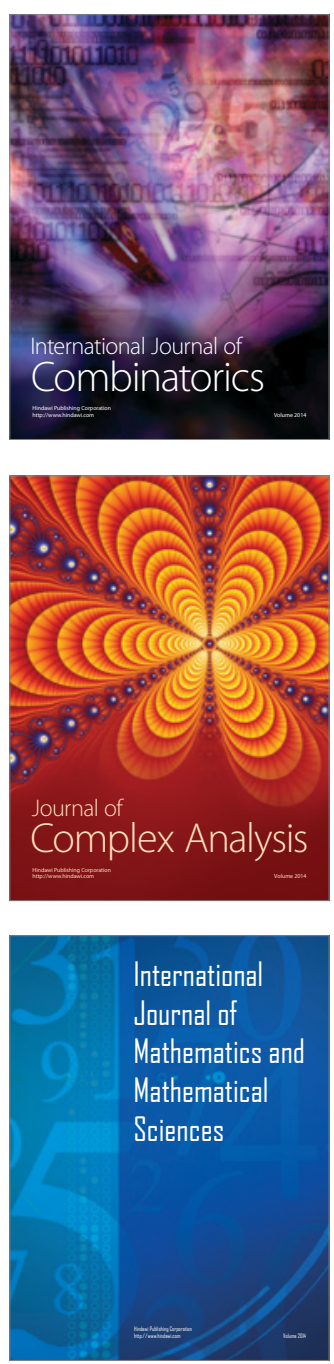
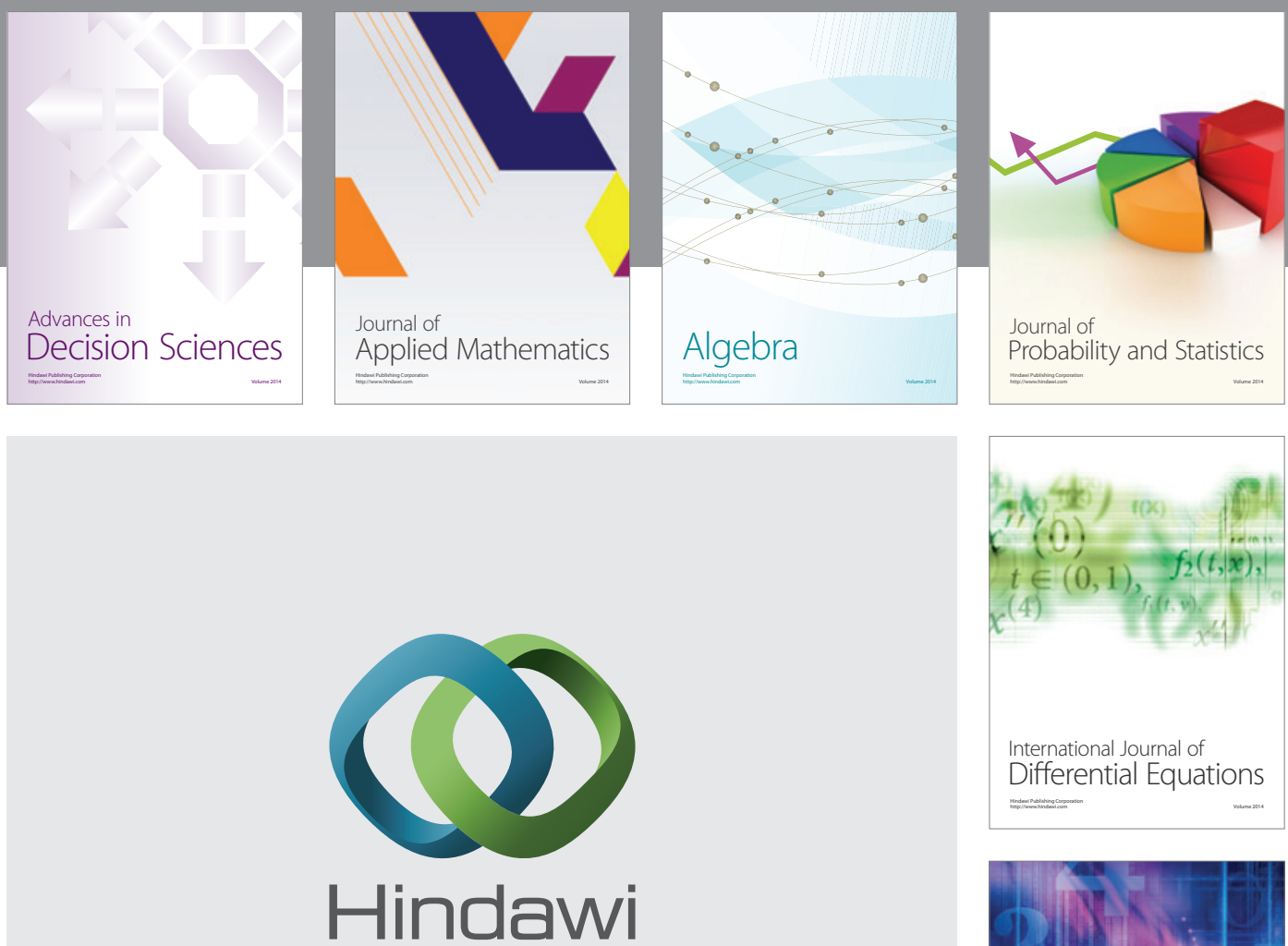

Submit your manuscripts at http://www.hindawi.com
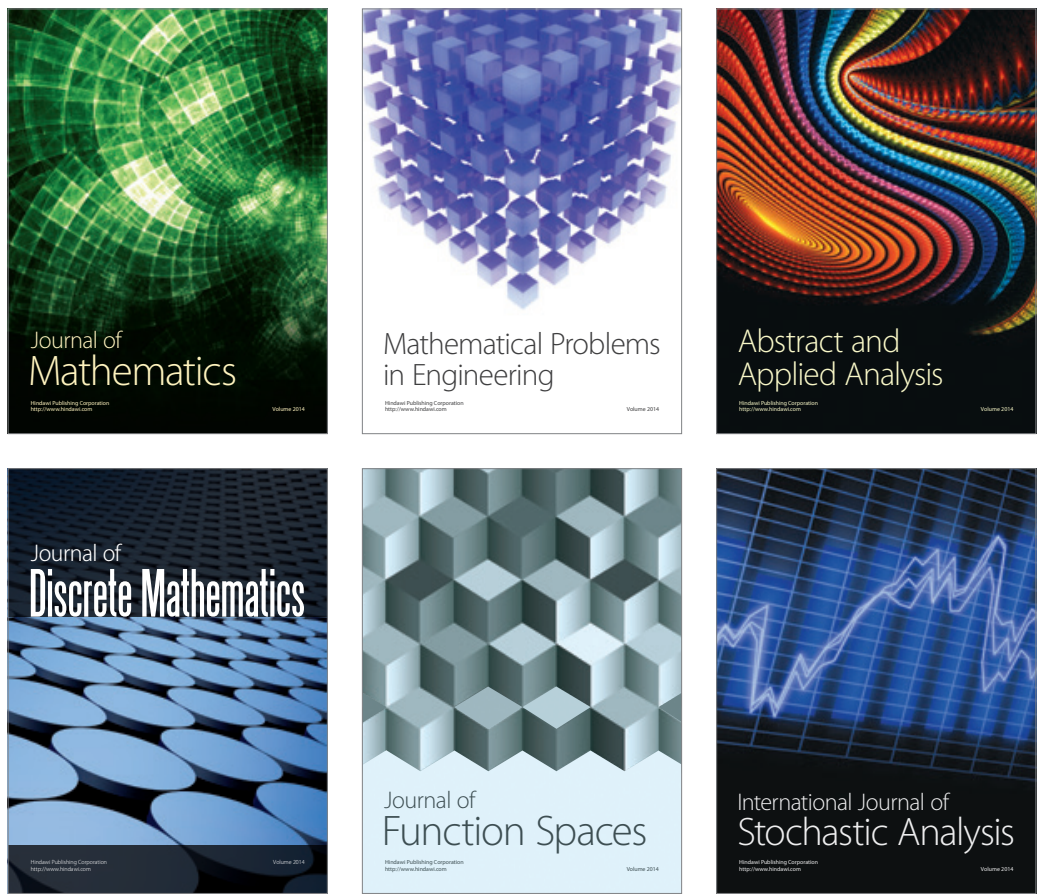

Journal of

Function Spaces

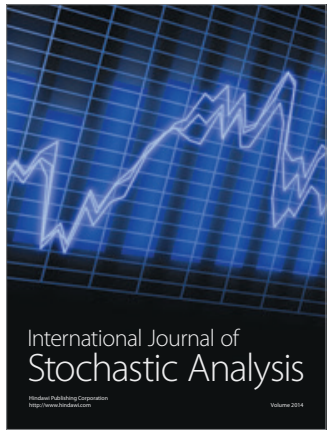

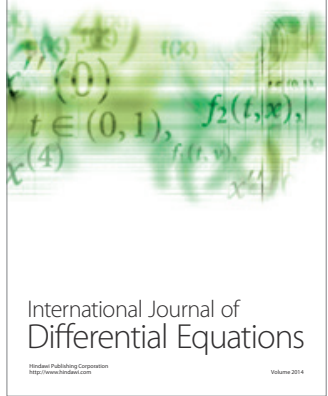
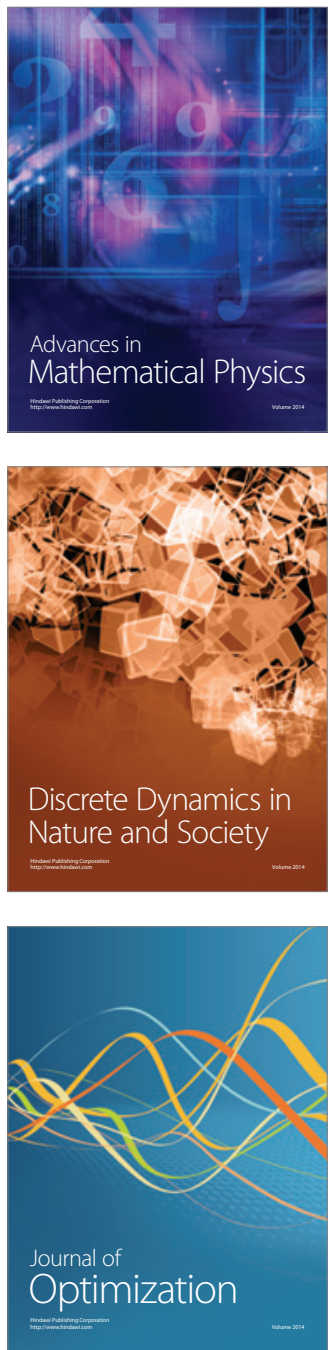